\title{
Problem of Determining the Two-Dimensional Absorption Coefficient in a Hyperbolic-Type Equation
}

\author{
Durdimurat K. Durdiev \\ Bukhara State University, Bukhara, Uzbekistan \\ E-mail:durdiev65@mail.ru \\ Received March 25, 2010; revised May 16, 2010; accepted May 29, 2010
}

\begin{abstract}
The problem of determining the hyperbolic equation coefficient on two variables is considered. Some additional information is given by the trace of the direct problem solution on the hyperplane $x=0$. The theorems of local solvability and stability of the solution of the inverse problem are proved.
\end{abstract}

Keywords: Inverse Problem, Hyperbolic Equation, Delta Function, Local Solvability

\section{Statement of the Problem and the Main Results}

We consider the generalized Cauchy problem

$$
\begin{gathered}
u_{t t}-u_{x x}-b(x, t) u_{t}=\delta(x, t-s), \quad(x, t) \in R^{2}, s>0, \\
\left.u\right|_{t<0} \equiv 0,
\end{gathered}
$$

where $\delta(x, t)$ is the two-dimensional Dirac delta function, $b(x, t)$ is a continuous function, $s$ is a problem parameter, and $u(x, t, s)$. We pose the inverse problem as follows: it is required to find absorption coefficient $b(x, t)$ if the values of the solution for are known, i.e., if the function

$$
u(0, t, s)=f(t, s), t>0, s>0 .
$$

Definition. A function $b(x, t)$ such that the solution of problem (1) corresponding to this function satisfies relation (2) is called a solution of inverse problem (1), (2).

The inverse problem posed in this paper is two-dimensional. For the case where $b(x, t)=b(x)$ the solvability problems for different statements of problems close to (1), (2) were studied in [1] (Chapter 2) and [2] (Chapter 1). The solvability problems for multidimensional inverse problems were considered in [2] (Chapter $3),[3,4]$, where the local existence theorems were proved in the class of functions smooth one of the variables and analytic in the other variables. In [5], the problems of stability and global uniqueness were investigated for inverse problem of determining the nonstationary potential in hyperbolic-type equation. In this paper, we prove the local solvability theorem and stability of the solution of the inverse problem (1), (2).

Let

$$
\begin{gathered}
Q_{T}:=\{(t, s) \mid 0 \leq s \leq t \leq T\}, \\
\Omega_{T}:=\{(x, t)|0 \leq| x|\leq t \leq T-| x \mid\}, T>0,
\end{gathered}
$$

$C_{t}^{1}\left(Q_{T}\right)$ is the class of function continuous in $s$, continuously differentiable in $t$, and defined on $Q_{T}$. We let $B$ denote the set of function $b(x, t)$ such that

$$
b(x, t) \in C\left(\Omega_{T}\right), b(-x, t)=b(x, t) .
$$

Theorem 1. If at a $T>0 \quad f(t, s) \in C^{1}\left(Q_{T}\right)$ and the condition

$$
f(s+0, s)=\frac{1}{2}
$$

is met, then for all $T \in\left(0, T_{-} 0\right), T_{0}=(1 / 40) \alpha_{0}, \quad \alpha_{0}=$ $4\left\|f_{t}^{\prime}(t, s)\right\|_{C\left(Q_{T}\right)}$ the solution to the inverse problems (1), (2) in the class of function $b(x, t) \in B$ exists and is unique.

Theorem 2. Let the conditions in Theorem 1 hold for the functions $f_{k}(t, s), k=1,2$, and let $b_{k}(x, t), k=1,2$, be the solutions to the inverse problems with the data $f_{k}(t, s), k=1,2$, respectively. Then the following estimate is valid for $T \in\left(0, T_{0}\right),\left(\left(T_{0}\right)\right.$ is defined in the same way as in proof of the Theorem 1)

$$
\left\|b_{1}(x, t)-b_{2}(x, t)\right\|_{C\left(\Omega_{T}\right)} \leq \frac{4}{1-\rho}\left\|f_{1}(t, s)-f_{2}(t, s)\right\|_{C_{t}^{1}\left(Q_{T}\right)},
$$


where $\rho=\frac{T}{T_{0}}$.

\section{Construction of a System Integral Equations for Equivalent Inverse Problems}

We represent the solution of problem (1) as

$$
u(x, t, s)=\frac{1}{2} \theta(t-s-|x|)+v(x, t, s) .
$$

where $\theta(t)=1$ for $t \geq 0, \theta(t)=0$, for $t<0, v(x$, $t, s)$ is a some regular function.

We substitute the Expression (5) in (1), take into account that $\theta(t-s-|x|) / 2$ satisfies (in the generalized sense) the equation $u_{t t}-u_{x x}=\delta(x) \delta^{\prime}(t-s)$, and obtain the problem for the function $v$ :

$$
\begin{aligned}
v_{t t}-v_{x x}= & b(x, t)\left[\frac{1}{2} \delta(t-s-|x|)+v_{t}(x, t, s)\right], \\
& (x, t) \in R^{2}, s>0, \\
\left.v\right|_{t, 0} \equiv 0 . &
\end{aligned}
$$

It follows from the d'Alembert formula that the solution of problem (6) satisfies the integral equation

$$
\begin{aligned}
v(x, t, s)= & \frac{1}{2} \iint_{\Delta(x, t)} b(\xi, \tau)\left[\frac{1}{2} \delta(\tau-s-|\xi|)+v_{t}(\xi, \tau, s)\right] . \\
& d \xi d \tau,(x, t) \in R^{2}, \quad s>0,
\end{aligned}
$$

where $\Delta(x, t)=\{(\xi, \tau)|0 \leq \tau \leq t-| x-\xi \mid, x-t \leq \xi \leq x+t\}$. We use the properties of the $\delta$ - function and easily obtain the relation in a different form:

$$
\begin{aligned}
v(x, t, s)= & \frac{1}{4} \int_{\frac{x+(t-s)}{2}}^{\frac{x-(t-s)}{2}} b(\xi, s+|\xi|) d \xi \\
& +\frac{1}{2} \iint_{Y(x, t, s)} b(\xi, \tau) v_{t}(\xi, \tau, s) d \tau d \xi \\
& t-s \geq|x|
\end{aligned}
$$

where the domain $Y(x, t, s)$ is defined by

$$
\begin{array}{r}
Y(x, t, s)=\left\{(\xi, \tau)|\xi|+s \leq \tau \leq t-|x-\xi|, \frac{x-(t-s)}{2}\right. \\
\left.\leq \xi \leq \frac{x+t-s}{2}, 0 \leq s \leq t, s=\text { const }\right\} .
\end{array}
$$

By differentiating the equality (8), we obtain

$$
\begin{aligned}
& v_{t}(x, t, s)=\frac{1}{8}[ b\left(\frac{x+t-s}{2}, \frac{x+t+s}{2}\right) \\
&\left.+b\left(\frac{x-t+s}{2}, \frac{-x+t+s}{2}\right)\right] \\
& \frac{1}{2} \int_{\frac{x-(t-s)}{2}}^{\frac{x+(t-s)}{2}} b(\xi, t-|x-\xi|) v_{t}(\xi, t-|x-\xi|, s) d \xi, t-s \geq|x| .
\end{aligned}
$$

It is obvious that $f(t, s)=u(0, t, s)=\frac{1}{2}+v(0, t, s)$ for $t \geq 0$. Moreover, the function $f(t, s)$ be must satisfy the condition (9).

We set $x=0$ in the equality (9), use the fact that the function $b(x, t)$ is even in $x$, and obtain the relation

$$
\begin{aligned}
f_{t}(t, s)= & \frac{1}{4} b\left(\frac{t-s}{2}, \frac{t+s}{2}\right) \\
& +\int_{-\frac{t-s}{2}}^{\frac{t-s}{2}} b(\xi, t-\xi) v_{t}(\xi, t-\xi, s) d \xi, \\
& (t, s) \in Q_{T} .
\end{aligned}
$$

We rewrite this equality, replacing $(t-s) / 2$ with $|x|$ and $(t+s) / 2$ with $t$, and solve it for $b(x, t)$. We obtain

$$
\begin{aligned}
b(x, t)= & 4 f_{t}^{\prime}(t+|x|, t-|x|)-4 \int_{-|x|}^{|x|} b(\xi, t+|x|-\xi) . \\
& v_{t}(\xi, t+|x|-\xi, t-|x|) d \xi, \quad t \geq|x| .
\end{aligned}
$$

Let

$$
Y_{T}=\{(x, t, s)|| x|+s \leq t \leq T-| x \mid, 0 \leq s \leq t \leq T\}
$$

The domain $Y_{T}$ in the space of the variables $x, t$, and $s$ is a pyramid with the base $\Omega_{t}$ and vertex $(0, T, T / 2)$. To find the value of the function $b$ at $(x, t)$, it is hence necessary to integrate $b(x, t)$ over the interval with the endpoints $(-|x|, t)$ and $(|x|, t)$ and to integrate the function $v_{t}(x, t, s)$ over the interval with the endpoints $(-|x|, t, t-|x|)$ and $(|x|, t, t-|x|)$, which belong to the domain $Y_{T}$.

One can rewrite the system of Equations (9) and (10) in the nonlinear operator form,

$$
\psi=\mathrm{A} \psi,
$$

where 


$$
\begin{aligned}
\psi=\left[\begin{array}{l}
\psi_{1}(x, t, s) \\
\psi_{2}(x, t)
\end{array}\right]= & {\left[v_{t}(x, t, s)-\frac{1}{8}\left[b\left(\frac{x+t-s}{2}, \frac{x+t+s}{2}\right)\right.\right.} \\
& \left.\left.+b\left(\frac{x-t+s}{2}, \frac{-x+t+s}{2}\right)\right]\right] \\
& b(x, t)
\end{aligned}
$$

The operator $A$ is defined on the set of functions $\psi \in C\left[Y_{T}\right]$ and, according to (9), (10), has the form

$$
A=\left(A_{1}, A_{2}\right)
$$

where

$$
\begin{gathered}
A_{1} \psi=\frac{1}{2} \int_{\frac{x-(t-s)}{2}}^{\frac{x+(t-s)}{2}} \psi_{2}(\xi, t-|x-\xi|)\left\{\psi_{1}(\xi, t-|x-\xi|)\right. \\
+\frac{1}{8}\left[\psi_{2}\left(\frac{\xi+t-|x-\xi|-s}{2}, \frac{\xi+t-|x-\xi|+s}{2}\right)\right. \\
\left.\left.+\psi_{2}\left(\frac{\xi-t+|x-\xi|+s}{2}, \frac{-\xi+t-|x-\xi|+s}{2}\right)\right]\right\} d \xi \\
A_{2} \psi=4 f_{t}^{\prime}(t+|x|, t-|x|)-4 \int_{-|x|}^{|x|} \psi_{2}(\xi, t+|x|-\xi) . \\
\left\{\psi_{1}(\xi, t+|x|-\xi, t-|x|)+\frac{1}{8}\left[\psi_{2}(|x|, t)\right.\right. \\
\left.\left.+\psi_{2}(\xi-|x|, t-\xi)\right]\right\} \mathrm{d} \xi .
\end{gathered}
$$

At fulfillment of the condition (3) the inverse problem $(1),(2)$ is equivalent to the operator Equation (11).

\section{Proofs of the Theorems}

Define

$$
\|\psi\|_{T}=\max \left(\left\|\psi_{1}\right\|_{C\left(Y_{T}\right)},\left\|\psi_{2}\right\|_{C\left(\Omega_{T}\right)}\right) .
$$

Let $\mathrm{s}$ be the set of $\psi \in C\left(Y_{T}\right)\left(\Omega_{T} \subset Y_{T}\right)$ that satisfy the following conditions:

$$
\left\|\psi-\psi^{0}\right\|_{T} \leq\left\|\psi^{0}\right\|_{T},
$$

where $\psi^{0}=\left(\psi_{01}, \psi_{02}\right)=\left(0,4 f_{t}^{\prime}(t+|x|, t-|x|)\right.$. It is obviously, that $\left\|\psi^{0}\right\|_{T} \leq 4\left\|f_{t}^{\prime}(t, s)\right\|_{C\left(Q_{T}\right)}=\alpha_{0}\left(Q_{T} \subset Y_{T}\right)$. Now we can show that if $T$ is small enough, $A$ is a contraction mapping operator in $S$. The local theorem of existence and uniqueness then follows immediately from the contraction mapping principle. First let us prove that $A$ has the first property of a contraction mapping operator, i.e., if $\psi \in S$, then $A \psi \in S$ when $T$ is small enough. Let $\psi \in S$. It is then easy to see that

$$
\|\psi\|_{T} \leq\left\|\psi-\psi^{0}\right\|_{T}+\left\|\psi^{0}\right\|_{T} \leq 2 \alpha_{0} .
$$

Furthermore, one has

$$
\begin{aligned}
& \left|A_{1} \psi-\psi_{01}\right| \leq \frac{1}{2} \int_{\frac{x-(t+s)}{2}}^{\frac{x+(t+s)}{2}}\left|\psi_{2}(\xi, t-|x-\xi|)\right| \\
& \times\left\{|\psi(\xi, t-|x-\xi|, s)|+\frac{1}{8}\left[\mid \psi_{2}\left(\frac{\xi+t-|x-\xi|-s}{2},\right.\right.\right. \\
& \left.\frac{\xi+t-|x-\xi|+s) \mid}{2}\right) \mid \psi_{2}\left(\frac{\xi-t+|x-\xi|+s}{2},\right. \\
& \frac{-\xi+t-|x-\xi|+s)||}{2} d \xi \leq\left.\frac{5 T}{8} \alpha_{0}|| \psi^{0}\right|_{T} ^{2}, \\
& \left|A_{2} \psi^{2}-\psi_{02}\right| \leq 4 \int_{-|x|}\left|\psi \psi_{2}(\xi \sigma, t+|x|-\xi)\right| \\
& \times\left\{\left|\psi_{1}(\xi, t+|x|-\xi, t-|x|)\right|+\frac{1}{8}\left[\left|\psi_{2}(|x|, t)\right|\right.\right. \\
& \left.\left.+\left|\psi_{2}(\xi-|x|, t-\xi)\right|\right]\right\} d \xi \leq\left. 10 T \alpha_{0}|| \psi^{0}\right|_{T} ^{2} .
\end{aligned}
$$

Therefore, if $T^{*}=1 / 10 \alpha_{0}$, then for $T \in\left(0, T_{0}\right)$ the operator A satisfies the condition $A \psi \in S$. Consider next the second property of contraction mapping operator for A i.e., if $\psi^{(1)} \in S, \quad \psi^{(2)} \in S$, then $\left\|A \psi^{(1)}-A \psi^{(1)}\right\|$ $\leq \rho\left\|\psi^{(1)}-\psi^{(1)}\right\|$ with $\rho<1$, when $T$ is small enough. Let $\psi^{(1)} \in S, \psi^{(2)} \in S$. Then one has

$$
\left|A_{1} \psi^{(1)}-A_{1} \psi^{(2)}\right| \leq\left.\frac{1}{2}\right|_{\frac{x-(t+s)}{2}} ^{\frac{x+(t+s)}{2}}\left\{\left(\psi_{2}^{(1)}-\psi_{2}{ }^{(2)}\right)(\xi, t-|x-\xi|)\right.
$$

$\left\{\psi_{2}^{(1)}(\xi, t-|x-\xi|, s)+\frac{1}{8}\left[\psi^{(1)}\right)_{2}\left(\frac{\xi+t-|x-\xi|-s}{2}\right.\right.$,

$\left.\frac{\xi+t-|x-\xi|+s}{2}\right)+\psi^{(1)} 2\left(\frac{\xi-t+|x-\xi|+s}{2}\right.$,

$\left.\left.\left.\frac{-\xi+t-|x-\xi|+s}{2}\right)\right]\right\}+\psi_{2}^{(2)}(\xi, t-|x-\xi|, s)$

$\times\left\{\left(\psi_{1}{ }^{(1)}-\psi_{1}{ }^{(2)}\right)(\xi, t-|x-\xi|, s)+\frac{1}{8}\left[\left(\psi_{2}{ }^{(1)}-\psi_{2}{ }^{(2)}\right)\right.\right.$

$\left(\frac{\xi+t-|x-\xi|-s}{2}, \frac{\xi+t-|x-\xi|+s}{2}\right)$ 


$$
\begin{aligned}
& +\left(\psi_{2}^{(1)}-\psi_{2}^{(2)}\right) \\
& \left.\left.\left(\frac{\xi-t+|x-\xi|+s}{2}, \frac{-\xi+t-|x-\xi|+s}{2}\right)\right]\right\} \\
& d \xi\left|\leq \frac{5 T}{2} \alpha_{0}\right|\left|\psi^{(1)}-\psi^{(2)}\right|_{T}, \\
& \left|A_{2} \psi^{(1)}-A_{2} \psi^{(2)}\right| \leq 4 \mid \int_{-|x|}^{|x|}\left\{\left(\psi_{2}^{(1)}-\psi_{2}^{(2)}\right)\right. \\
& (\xi, t+|x|-\xi)\left\{\psi_{1}^{(1)}(\xi, t+|x|-\xi, t-|x|)\right. \\
& \left.+\frac{1}{8}\left[\psi_{2}^{(1)}(|x|, t)+\psi_{2}^{(1)}(\xi-|x|, t-\xi)\right]\right\} \\
& +\psi(2)(\xi, t+|x|-\xi) \times\left\{\left(\psi \psi_{1}^{(1)}-\psi_{1}^{(2)}\right)\right. \\
& (\xi, t+|x|-\xi, t-|x|)+\frac{1}{8}\left[\left(\psi_{2}^{(1)}-\psi_{2}^{(1)}\right)\right. \\
& \left.\left.\left.(|x|, t)+\left(\psi_{2}^{(1)}-\psi_{2}^{(1)}\right)(\xi-|x|, t-\xi)\right]\right\}\right\} \\
& d \xi\left|\leq 40 T \alpha_{0}\right| \mid \psi^{(1)}-\psi^{(2)} \|_{T} \cdot
\end{aligned}
$$

It follows from the preceding estimates that if $T_{0}=1 / 40 \alpha_{0}$, then for $T \in\left(0, T_{0}\right)$ the operator $\mathrm{A}$ is a contraction operator with $\rho=T / T_{0}$ on the set $S$. Therefore, the Equation (11) has a unique solution which belongs to $S$ according to the contraction mapping principle. The solution is the limit of the sequence $\psi^{[n]}$, $n=0,1,2, \ldots, \quad$ where $\psi^{[0]}=\psi(0), \quad \psi^{[n+1]}=A \psi^{[n]}$, and the series

$$
\psi^{[0]}+\sum_{n=0}^{\infty}\left(\psi^{[n+1]}-\psi^{[n]}\right)
$$

converges not slower than the series

$$
\left\|\psi^{[0]}\right\|_{T}+\sum_{n=0}^{\infty} \rho^{n}\left\|\psi^{[1]}-\psi^{[0]}\right\|_{T}
$$

We now prove Theorem 2. Since the conditions Theorem 1 hold, the solution belong to the set $S$ and
$\left\|\psi_{i}\right\|_{T} \leq 2 \alpha_{0}, i=1,2$. Let $\psi^{(k)}, k=1,2 \quad$ be vector functions which are the solution of the Equation (11) with the data $f_{k}(t, s), k=1,2$, respectively, i.e.,

$$
\psi^{(k)}=A \psi^{(k)}
$$

From the previous results in the proof of Theorem 1, it follows that

$$
\begin{aligned}
& \left|\psi_{1}^{(k)}-\psi_{2}^{(k)}(x, t, s)\right| \leq 4\left\|f_{1}(t, s)-f_{2}(t, s)\right\|_{C_{t}^{1}\left(Q_{T}\right)} \\
& +40 T \alpha_{0}\left\|\psi_{1}-\psi_{2}\right\|_{T}, k=1,2 .
\end{aligned}
$$

Therefore, one has

$\left\|\psi_{1}-\psi_{2}\right\|_{T} \leq 4\left\|f_{1}(t, s)-f_{2}(t, s)\right\|_{C_{t}^{1}\left(Q_{T}\right)+\rho\left\|\psi_{1}-\psi_{2}\right\|_{T}}$

The last inequality gives

$$
\left\|\psi_{1}-\psi_{2}\right\|_{T} \leq \frac{4}{1-\rho}\left\|f_{1}(t, s)-f_{2}(t, s)\right\|_{C_{t}^{1}\left(Q_{T}\right)}
$$
(12).

The stability estimate (4) follows from the inequality

\section{References}

[1] V. G. Romanov, "Inverse Problems of Mathematical Physics," in Russian, Publishing House "Nauka", Moscow, 1984.

[2] V. G. Romanov, "Stability in Inverse Problems," in Russian, Nauchnyi Mir, Moscow, 2005.

[3] D. K. Durdiev, "A Multidimensional Inverse Problem for an Equation with Memory," Siberian Mathematical Journal, Vol. 35, No. 3, 1994, pp. 514-521.

[4] D. K. Durdiev, "Some Multidimensional Inverse Problems of Memory Determination in Hyperbolic Equations," Journal of Mathematical Physics, Analysis, Geometry, Vol. 3, No. 4, 2007, pp. 411-423.

[5] D. K. Durdiev, "Problem of Determining the Nonstationary Potential in a Hyperbolic-Type Equation," Journal of Theoretical and Mathematical Physics, Vol. 2, No. 156, 2008, pp. 1154-1158. 